\title{
Association of Fragmented QRS with Subclinical Left Ventricular Dysfunction in Patients with Obstructive Sleep Apnea
}

\author{
Adem Adar ${ }^{a}$ Abdulkadir Kırış ${ }^{c}$ Yılmaz Bülbüld Hüseyin Bektaş ${ }^{c}$ Murat Acat ${ }^{d}$ \\ Hasan Casim $^{\mathrm{b}}$ Orhan Onalan ${ }^{\mathrm{a}}$ \\ Departments of a Cardiology and ${ }^{b}$ Chest Disease, Faculty of Medicine, Karabuk University Hospital, Karabuk \\ University, Karabuk, and Departments of ${ }^{c}$ Cardiology and ${ }^{d}$ Chest Disease, Faculty of Medicine, Karadeniz Technical \\ University, Trabzon, Turkey
}

\section{Key Words}

Fragmented QRS · Obstructive sleep apnea .

Left ventricular dysfunction - Myocardial fibrosis

\begin{abstract}
Objective: We aimed to investigate whether fragmented QRS (fQRS) is associated with subclinical left ventricular (LV) dysfunction in patients with obstructive sleep apnea (OSA). Subjects and Methods: A total of 141 patients with OSA who had normal LV ejection fraction (LVEF) were included in the study. The fQRS was defined as the presence of an additional $R$ wave, notching of $R$ or $S$ wave or the presence of fragmentation in 2 contiguous electrocardiography (ECG) leads. Subclinical LV dysfunction was defined as the presence of a tissue Doppler-derived Tei index of $\geq 0.5$ in the absence of impaired LVEF $(<50 \%)$ as assessed by transthoracic echocardiography. Results: Of the 141 patients, 71 (50.4\%) had subclinical LV dysfunction. Overall, the prevalence of the fQRS was $61 \%(86 / 141)$. Patients with fQRS had significantly higher Tei indices than those without fQRS [median 0.66, interquartile range (IQR) 0.39 vs. median 0.40, IQR $0.15, \mathrm{p}<0.001$ ].
\end{abstract}

The presence of fQRS on ECG predicted subclinical LV dysfunction in univariate logistic regression analysis [odds ratio (OR) 6.69, 95\% confidence interval (Cl) 3.10-14.43]. The association remained significant after adjusting for all potential confounders (OR 4.59, 95\% Cl 1.94-10.87). Conclusion: fQRS on ECG was an independent predictor of subclinical LV dysfunction in patients with OSA. This simple tool might help to identify OSA patients who could be at risk for developing overt cardiac dysfunction.

(c) 2015 S. Karger AG, Basel

\section{Introduction}

Obstructive sleep apnea (OSA) is characterized by repetitive episodes of upper airway collapse during sleep that lead to apnea, hypopnea and intermittent hypoxia [1]. Patients with OSA are at increased risk for cardiovascular disease [2-4]. Cardiovascular events in OSA are related to the presence of myocardial involvement rather than OSA itself $[5,6]$. Myocardial fibrosis is one of the major myocardial structural alterations in patients with

\begin{tabular}{ll}
\hline KARGER 125/s & $\begin{array}{l}\text { ○ 2015 S. Karger AG, Basel } \\
1011-7571 / 15 / 0244-0376 \$ 39.50 / 0 \quad \text { Karger }\end{array}$ \\
E-Mail karger@karger.com & $\begin{array}{l}\text { This is an Open Access article licensed under the terms of the } \\
\text { Creative Commons Attribution-NonCommercial 3.0 Un- } \\
\text { ported license (CC BY-NC) (www.karger.com/OA-license), } \\
\text { applicable to the online version of the article only. Distribu- } \\
\text { tion permitted for non-commercial purposes only. }\end{array}$
\end{tabular}

Orhan Onalan, MD, Associate Professor

Department of Cardiology

Faculty of Medicine, Karabuk University Hospital

TR-78050 Karabuk (Turkey)

E-Mail orhanonalan@ karabuk.edu.tr 
OSA and may eventually lead to cardiac dysfunction. Hypoxia-induced sympathetic activity, sleep fragmentation, systemic inflammation, endothelial dysfunction, oxidative stress and metabolic abnormalities may promote myocardial fibrosis and left ventricular (LV) dysfunction in patients with OSA [6-13]. Fragmented QRS (fQRS) has been shown to be a marker of myocardial fibrosis or scar tissue in various clinical conditions [14-17]. To date, no study has evaluated the association between fQRS and LV dysfunction in patients with OSA. Hence, we investigated whether or not $\mathrm{fQRS}$ is associated with subclinical LV dysfunction in patients with OSA.

\section{Subjects and Methods}

\section{Patients}

All patients scheduled for an overnight polysomnographic study between June 2011 and January 2012 were approached to participate in this study. Patients diagnosed with OSA were included. Exclusion criteria were: (1) a history of coronary revascularization, (2) angiographic evidence of coronary artery disease, (3) a history of hospitalization for acute coronary syndromes, (4) a positive noninvasive test for myocardial ischemia, (5) resting electrocardiography (ECG) suggestive of myocardial ischemia or infraction, (6) a history of hospitalization for heart failure, (7) bundle branch block on ECG, (8) echocardiographic findings of reduced LV ejection fraction (LVEF) $<50 \%$, moderate-to-severe valve disease and wall motion abnormality and (9) morbid obesity. Overall, 219 patients were accepted to participate in the study; 176 (80.4\%) were diagnosed with OSA but 35 of these were then excluded based on the above exclusion criteria. Hence, 141 patients (95 males and 46 females) with OSA were included in the study. Diabetes was defined as being on treatment with insulin or oral antidiabetic drugs. Hypertension and hyperlipidemia were defined as being on antihypertensive or lipid-lowering drugs, respectively. The Institutional Ethics Committee approved the study protocol. Written informed consent was obtained from all patients.

\section{Assessment of $f Q R S$}

The fQRS was defined as the presence of an additional $\mathrm{R}$ wave $(R ')$, notching of R or $S$ wave or the presence of fragmentation of more than one R' in 2 contiguous ECG leads (filter range $0.15-100$ $\mathrm{Hz}, 25 \mathrm{~mm} / \mathrm{s}, 10 \mathrm{~mm} / \mathrm{mV}$ ). Two experienced cardiologists (O.O. and A.A.), blinded to the patients' data, evaluated all ECGs, and the interobserver agreement was excellent $[\mathrm{k}=0.93, \mathrm{p}<0.001,95 \%$ confidence interval (CI) 0.86-0.99] for the presence of fQRS on ECG.

\section{Polysomnography}

The diagnosis of OSA was based on polysomnographic study (Alice Sleepware; Philips Respironics, Inc., Murrysville, Pa., USA). All variables were recorded on a commercially available computer system, including electroencephalography (F3M2, F4M1, C3M2, C4M1, O1M2 and O2M1), bilateral electrooculography, submental electromyography, thoracic and abdominal movements measured by uncalibrated inductive plethysmography, saturation of oxyhemoglobin $\left(\mathrm{SaO}_{2}\right)$ using a finger oximeter, airflow through the nose and mouth recorded by thermistors, 2 contiguous ECG leads, a snoring microphone and video monitoring with an infrared video camera. The entire recording was observed by an experienced sleep technician. Apnea was defined as the continuous cessation of airflow for $>10 \mathrm{~s}$, and hypopnea was defined as a reduction in airflow of $\geq 30 \%$ lasting for $\geq 10$ s accompanied by a decrease in oxygen saturation $\left(\mathrm{SpO}_{2}\right)$ of at least $4 \%$ [18]. The length of time $\mathrm{SpO}_{2}$ was $<90 \%$ during sleep and the minimum $\mathrm{SaO}_{2}$ for each patient was calculated. Apnea-hypopnea index (AHI) values were calculated as the number of episodes of apnea and hypopnea per hour over the total sleep time. Patients with an AHI $\geq 5$ were considered to have OSA. The severity of OSA was classified according to the AHI (mild: $\geq 5$ and $<15$, moderate: $\geq 15$ and $<30$ and severe: 230) [19].

\section{Echocardiographic Examination}

All patients were examined in the left lateral decubitus position using a commercially available system (Vivid 7; GE Medical Systems, Horten, Norway) with a phased-array 3.5-MHz transducer and tissue Doppler imaging software. The conventional M-mode, $\mathrm{B}$-mode and Doppler parameters were measured according to the American Society of Echocardiography guidelines [20]. LV enddiastolic and end-systolic diameters and posterior and septal wall thicknesses were measured. LV mass (LVM) was calculated using the Devereux equation: $\mathrm{LVM}=0.8 \times[1.04(\mathrm{LVEDD}+\mathrm{IVST}+$ $\left.\mathrm{PWT}^{3}-\left(\mathrm{LVEDD}^{3}\right)\right]+0.6$, where LVEDD is LV end-diastolic diameter, IVST is intraventricular septal wall thickness and PWT is posterior wall thickness [21]. LVM index (LVMI) was calculated by dividing the LVM by the body surface area. LV hypertrophy was defined as LVMI $>115 \mathrm{~g} / \mathrm{m}^{2}$ for men and $>95 \mathrm{~g} / \mathrm{m}^{2}$ for women [22]. LVEF was measured using the modified biplane Simpson's rule. Tissue Doppler imaging was performed from the lateral mitral annulus. The time interval from the end to the onset of mitral annular velocity wave during diastole (a) and the duration of the peak systolic mitral annular velocity (b) were measured (all values were averaged over 3 consecutive cardiac cycles), and then the Tei index was calculated as isovolumetric contraction time plus isovolumetric relaxation time divided by ejection time, i.e. [ $(\mathrm{a}-\mathrm{b}) / \mathrm{b}][23,24]$. A Tei index of $\geq 0.5$ was considered abnormal. Subclinical LV dysfunction was defined as the presence of abnormal Tei index $(\geq 0.5)$ in the absence of impaired LVEF $(<50 \%)$.

\section{Statistical Analysis}

Continuous variables are expressed as mean \pm standard deviation (SD) or median and interquartile range (IQR), where appropriate. Categorical variables are presented as numbers and percentages. The distributions of the continuous variables across the study groups were tested with the Shapiro-Wilks test. Continuous data were analyzed using the Student $t$ test or the Mann-Whitney $\mathrm{U}$ test, and categorical data were compared using the $\chi^{2}$ test or the Fisher exact test. Multivariate logistic regression analyses were conducted to assess the association between $\mathrm{fQRS}$ and the presence of subclinical LV dysfunction. In multivariate regression models, effect size was adjusted for variables with a significance level $\leq 0.10$ in the univariate analysis. Adjusted odds ratios (ORs) and their corresponding CIs were presented. A 2 -tailed $\mathrm{p}$ value $<0.05$ was considered statistically significant. All statistical analyses were performed using the IBM SPSS software (IBM SPSS Statistics for Windows, v21.0; IBM Corp., Armonk, N.Y., USA). 
Table 1. Baseline characteristics of the study groups

\begin{tabular}{|c|c|c|c|}
\hline & \multicolumn{2}{|c|}{ Subclinical LV dysfunction } & \multirow[t]{2}{*}{$\mathrm{p}$ value } \\
\hline & yes $(n=71)$ & no $(\mathrm{n}=70)$ & \\
\hline Age, years & $52 \pm 11$ & $48 \pm 8$ & 0.031 \\
\hline Males & $49(69 \%)$ & $46(66 \%)$ & 0.676 \\
\hline Body surface area, $\mathrm{m}^{2}$ & $2.05 \pm 0.19$ & $2.06 \pm 0.17$ & 0.916 \\
\hline Body mass index & $31 \pm 5$ & $32 \pm 5$ & 0.666 \\
\hline Systolic blood pressure, $\mathrm{mm} \mathrm{Hg}$ & $128 \pm 22$ & $129 \pm 20$ & 0.773 \\
\hline Diastolic blood pressure, $\mathrm{mm} \mathrm{Hg}$ & $75 \pm 16$ & $76 \pm 13$ & 0.586 \\
\hline Hypertension & $26(37 \%)$ & $27(39 \%)$ & 0.811 \\
\hline Diabetes & $11(15 \%)$ & $11(16 \%)$ & 0.971 \\
\hline Hyperlipidemia & $16(23 \%)$ & $16(23 \%)$ & 0.964 \\
\hline Smoking & $21(30 \%)$ & $19(27 \%)$ & 0.480 \\
\hline Obesity & $39(55 \%)$ & $43(63 \%)$ & 0.320 \\
\hline Total cholesterol, mg/dl & $185(71)$ & $189(52)$ & 0.571 \\
\hline Triglycerides, mg/dl & $151(102)$ & $151(105)$ & 0.988 \\
\hline $\mathrm{LDL}, \mathrm{mg} / \mathrm{dl}$ & $112(52)$ & $105(49)$ & 0.981 \\
\hline $\mathrm{HDL}, \mathrm{mg} / \mathrm{dl}$ & 45 (19) & $43(16)$ & 0.789 \\
\hline AHI, events/h & $34.5(31)$ & $21.7(25)$ & 0.002 \\
\hline Lowest nocturnal $\mathrm{SpO}_{2}$ & $81(14)$ & $80(23)$ & 0.372 \\
\hline Length of time spent in $\mathrm{SpO}_{2}<90 \%$, min & $14(69)$ & $5(27)$ & 0.061 \\
\hline Sleeping time, $\min$ & $354.5(48)$ & $364.5(86)$ & 0.610 \\
\hline \multicolumn{4}{|l|}{ OSA severity } \\
\hline Mild & $11(15 \%)$ & $24(34 \%)$ & 0.034 \\
\hline Moderate & $18(25 \%)$ & $15(21 \%)$ & \\
\hline Severe & $42(59 \%)$ & $31(44 \%)$ & \\
\hline ACE inhibitor & $17(24 \%)$ & $25(36 \%)$ & 0.126 \\
\hline$\beta$-Blocker & $11(15 \%)$ & $8(11 \%)$ & 0.480 \\
\hline LVEDd, mm & $48(6)$ & $46(3)$ & 0.190 \\
\hline IVST, mm & $10(1)$ & $10(1)$ & 0.996 \\
\hline PWT, mm & $10(1)$ & $10(1)$ & 0.620 \\
\hline LVEF, \% & $65(3)$ & $65(8)$ & 0.051 \\
\hline Left atrial diameter, $\mathrm{mm}$ & $39(3)$ & $36(6)$ & $<0.001$ \\
\hline LVM, g & $176(42)$ & $170(49)$ & 0.171 \\
\hline LVMI, g/m² & $85(22)$ & $82(24)$ & 0.116 \\
\hline LV hypertrophy & $12(17 \%)$ & $9(13 \%)$ & 0.546 \\
\hline fQRS & $58(82 \%)$ & $28(40 \%)$ & $<0.0001$ \\
\hline
\end{tabular}

Continuous variables are presented as mean $\pm \mathrm{SD}$ or median and interquartile range. Categorical variables are presented as $\mathrm{n}(\%)$. ACE = Angiotensin-converting enzyme; HDL = high-density lipoprotein; IVST = interventricular septal thickness; LDL = low-density lipoprotein; LVEDd = LV end-diastolic diameter; PWT = posterior wall thickness.

\section{Results}

The mean age of the study population was $50 \pm 10$ years. Of the 141 patients with OSA, 71 (50.4\%) had subclinical LV dysfunction. The demographic, clinical and echocardiographic characteristics of the patients with and without subclinical LV dysfunction are given in table 1. Overall, the prevalence of the fQRS was $61 \%(86 / 141)$ in the study group. Patients with fQRS had significantly higher Tei index values than patients without fQRS (me- dian 0.66, IQR 0.39 vs. median 0.40, IQR 0.15, p < 0.001; fig. 1). Patients with subclinical LV dysfunction were older ( $52 \pm 11$ vs. $48 \pm 8$ years, $\mathrm{p}=0.031)$, had a higher prevalence of fQRS ( 82 vs. $40 \%, \mathrm{p}<0.0001$ ), higher AHI values (median 34.5, IQR 31 vs. median 21.7, IQR 25.1, p = $0.002)$, more severe OSA $(\mathrm{p}=0.034)$ and a larger left atrium (median 39, IQR 3 vs. median 36, IQR 6, p < 0.001) than patients without subclinical LV dysfunction. The study groups were similar with respect to all other demographic, clinical, echocardiographic and laboratory pa- 
Table 2. Univariate and multivariate analysis for subclinical LV dysfunction

\begin{tabular}{|c|c|c|c|c|}
\hline & \multicolumn{2}{|c|}{ Univariate analysis } & \multicolumn{2}{|c|}{ Multivariate analysis } \\
\hline & OR & $95 \% \mathrm{CI}$ & OR & $95 \%$ CI \\
\hline \multicolumn{5}{|l|}{ Model A } \\
\hline Age & 1.040 & $1.003-1.078$ & 1.025 & $0.981-1.071$ \\
\hline \multicolumn{5}{|l|}{ OSA severity } \\
\hline Mild & \multicolumn{4}{|c|}{ Reference category } \\
\hline Moderate & 2.62 & $0.97-7.04$ & 1.23 & $0.36-4.16$ \\
\hline Severe & 2.96 & $1.26-6.92$ & 0.94 & $0.29-3.07$ \\
\hline Length of time spent in $\mathrm{SpO}_{2}<90 \%$ & 1.009 & $1.001-1.016$ & 1.006 & $0.997-1.016$ \\
\hline LVEF & 0.933 & $0.870-1.000$ & 0.963 & $878-1.057$ \\
\hline Left atrial diameter & 0.996 & $0.983-1.009$ & 0.993 & $0.976-1.010$ \\
\hline fQRS & 6.69 & $3.10-14.43$ & 4.77 & $1.95-11.65$ \\
\hline \multicolumn{5}{|l|}{ Model B } \\
\hline Age & 1.040 & $1.003-1.078$ & 1.027 & $0.982-1.073$ \\
\hline AHI & 1.022 & $1.005-1.039$ & 1.009 & $0.986-1.032$ \\
\hline Length of time spent in $\mathrm{SpO}_{2}<90 \%$ & 1.009 & $1.001-1.016$ & 1.004 & $0.995-1.014$ \\
\hline LVEF & 0.933 & $0.870-1.000$ & 0.976 & $0.891-1.068$ \\
\hline Left atrial diameter & 0.996 & $0.983-1.009$ & 0.992 & $0.975-1.010$ \\
\hline fQRS & 6.69 & $3.10-14.43$ & 4.59 & $1.94-10.87$ \\
\hline
\end{tabular}

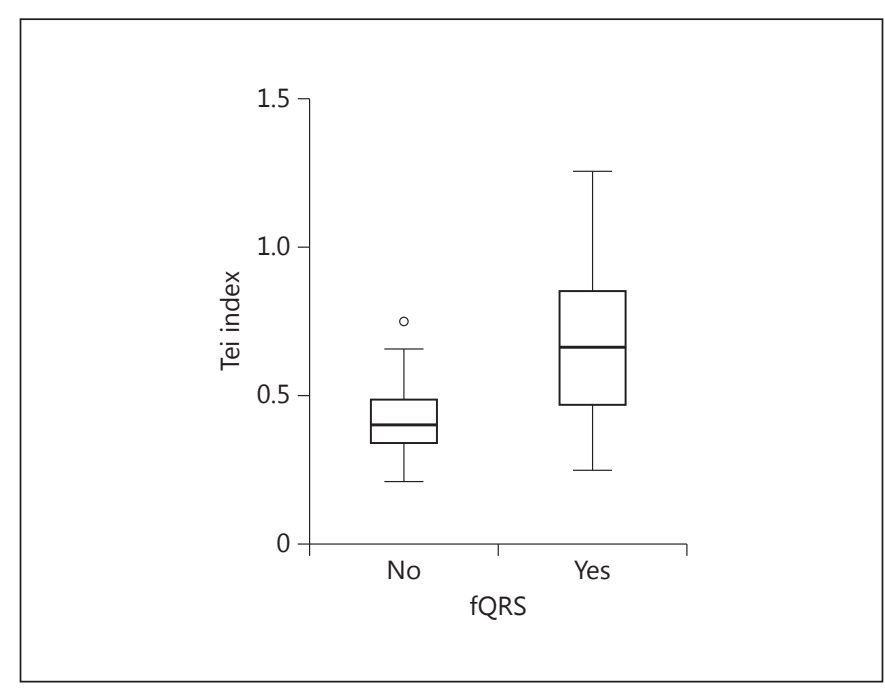

Fig. 1. Distribution of Tei index across the study groups.

rameters (table 1). The presence of fQRS on ECG predicted subclinical LV dysfunction in the univariate logistic regression analysis (OR 6.69, 95\% CI 3.10-14.43; table 2). The association remained significant after adjusting for all potential confounders (OR 4.77, 95\% CI 1.95-11.65) in the multivariate logistic regression analysis (table 2). Excluding OSA severity and retesting the
AHI using a different multivariate regression model (model B) did not change the results (OR 4.59, 95\% CI 1.94-10.87). The association of fQRS with subclinical LV dysfunction remained significant after adjusting for LVMI (model A: OR 6.35, 95\% CI 2.38-16.90 and model B: OR 5.88, 95\% CI 2.30-15.03) and blood pressure values (model A: OR 4.73, 95\% CI 1.90-11.80 and model B: OR $4.53,95 \%$ CI 1.87-10.99). Neither LVMI nor blood pressure values were associated with subclinical LV dysfunction in the multivariate analysis.

\section{Discussion}

In this study, the overall prevalence of fQRS was $61 \%$ in OSA patients who had a preserved LVEF. Patients with subclinical LV dysfunction had a significantly higher prevalence of fQRS on ECG than patients without subclinical LV dysfunction. Moreover, fQRS was independently associated with subclinical LV dysfunction in patients with OSA.

Patients with OSA are at an increased risk for clinical and subclinical cardiovascular disease [2-4]. Cardiovascular events in OSA are related to the presence of myocardial involvement rather than to OSA itself $[5,6]$, with fibrosis being one of the main myocardial structural changes reported in patients. Multiple mechanisms may 
contribute to the development of myocardial fibrosis in OSA patients, including hypoxia-induced sympathetic activity, sleep fragmentation, systemic inflammation, endothelial dysfunction, oxidative stress and metabolic abnormalities [6-13]. Several studies have reported a strong association between fQRS detected on ECG and myocardial fibrosis as assessed by gadolinium-enhanced cardiac magnetic resonance imaging $[14,16,17]$. To date, few studies have evaluated the association of fQRS with LV functions.

Canga et al. [25] found an association between fQRS and LV systolic and diastolic dysfunction in 259 patients who were admitted to their outpatient clinic. Yan et al. [26] studied the relationship between fQRS and LV functions, as assessed by speckle-tracking echocardiography, in 176 patients with coronary heart disease and preserved LVEF. They found fQRS to be associated with subclinical global and regional LV dysfunction and adverse cardiac events. Consistent with this, we found an independent association between fQRS and subclinical LV dysfunction in patients with OSA. We think that subclinical LV dysfunction in OSA patients is a consequence of myocardial structural changes, mainly myocardial fibrosis.

Myocardial fibrosis can be detected by histopathological evaluation, cardiac magnetic resonance imaging and scintigraphic methods. However, these imaging modalities and histopathological tests are not readily available, are impractical in some cases and also expensive. Myocardial fibrosis may disrupt QRS morphology and lead to fQRS on 12-lead ECG. Accordingly, the prevalence of fQRS is relatively low in the healthy general population [27] and relatively high in patients with myocardial fibrosis [14-17].

We evaluated subclinical LV dysfunction by means of the Tei index, which is an easily measured Doppler-de- rived parameter of global cardiac functions that allows evaluation of both systolic and diastolic ventricular performance [28]. A mean Tei index value of $>0.40$ for the left ventricle is considered abnormal in adults. It is a reproducible parameter and is not affected by blood pressure, heart rate, ventricular geometry, valvular insufficiencies or preload and afterload in patients who are in a supine position [28]. Accordingly, it has been found to be a useful tool for evaluating global cardiac performance in congestive heart failure, valvular heart disease, coronary heart disease and heart transplant patients [29]. Moreover, it provides prognostic information for patients with dilated cardiomyopathy, cardiac amyloidosis, pulmonary hypertension and coronary heart disease [29].

The limitations of this study were that myocardial structural changes were not shown with imaging modalities and the study sample was small.

\section{Conclusion}

fQRS on ECG is an independent predictor of subclinical LV dysfunction in patients with OSA. It is an inexpensive, widely available tool and could be used for noninvasive and indirect assessment of myocardial structural changes, mainly myocardial fibrosis, in patients with OSA. This simple tool might help to identify patients who are at risk of developing overt cardiac dysfunction.

\section{Disclosure Statement}

The authors have no conflicts of interest to disclose.

\section{References}

1 Epstein LJ, Kristo D, Strollo PJ Jr, et al: Clinical guideline for the evaluation, management and long-term care of obstructive sleep apnea in adults. J Clin Sleep Med 2009;5:263-276.

$\checkmark 2$ Ge X, Han F, Huang Y, et al: Is obstructive sleep apnea associated with cardiovascular and all-cause mortality? PLoS One 2013;8: e69432.

- 3 Dong JY, Zhang YH, Qin LQ: Obstructive sleep apnea and cardiovascular risk: metaanalysis of prospective cohort studies. Atherosclerosis 2013;229:489-495.

4 Kohli P, Balachandran JS, Malhotra A: Obstructive sleep apnea and the risk for cardiovascular disease. Curr Atheroscler Rep 2011; 13:138-146.
5 McNicholas WT, Bonsigore MR; Management Committee of ECAB: Sleep apnoea as an independent risk factor for cardiovascular disease: current evidence, basic mechanisms and research priorities. Eur Respir J 2007;29: 156-178.

-6 Baguet JP, Barone-Rochette G, Tamisier R, et al: Mechanisms of cardiac dysfunction in obstructive sleep apnea. Nat Rev Cardiol 2012;9: 679-688.

7 Lavie L: Oxidative stress inflammation and endothelial dysfunction in obstructive sleep apnea. Front Biosci (Elite Ed) 2012;4:13911403.

8 Gozal D, Kheirandish-Gozal L: Cardiovascular morbidity in obstructive sleep apnea: oxi- dative stress, inflammation, and much more. Am J Respir Crit Care Med 2008;177:369375.

9 O'Driscoll DM, Horne RS, Davey MJ, et al: Increased sympathetic activity in children with obstructive sleep apnea: cardiovascular implications. Sleep Med 2011;12:483-488.

10 Fletcher EC: Cardiovascular consequences of obstructive sleep apnea: experimental hypoxia and sympathetic activity. Sleep 2000; 23(suppl 4):S127-S131.

11 Seif F, Patel SR, Walia H, et al: Association between obstructive sleep apnea severity and endothelial dysfunction in an increased background of cardiovascular burden. J Sleep Res 2013;22:443-451. 
12 Zamarron C, Valdes Cuadrado L, Alvarez-Sala R: Pathophysiologic mechanisms of cardiovascular disease in obstructive sleep apnea syndrome. Pulm Med 2013;2013:521087.

13 Somers VK, Dyken ME, Clary MP, et al: Sympathetic neural mechanisms in obstructive sleep apnea. J Clin Invest 1995;96:1897-1904.

14 Basaran Y, Tigen K, Karaahmet T, et al: Fragmented QRS complexes are associated with cardiac fibrosis and significant intraventricular systolic dyssynchrony in nonischemic dilated cardiomyopathy patients with a narrow QRS interval. Echocardiography 2011;28:6268.

15 Das MK, Suradi H, Maskoun W, et al: Fragmented wide QRS on a 12-lead ECG: a sign of myocardial scar and poor prognosis. Circ Arrhythm Electrophysiol 2008;1:258-268.

16 Park SJ, On YK, Kim JS, et al: Relation of fragmented QRS complex to right ventricular fibrosis detected by late gadolinium enhancement cardiac magnetic resonance in adults with repaired tetralogy of fallot. Am J Cardiol 2012;109:110-115.

17 Homsi M, Alsayed L, Safadi B, et al: Fragmented QRS complexes on 12-lead ECG: a marker of cardiac sarcoidosis as detected by gadolinium cardiac magnetic resonance imaging. Ann Noninvasive Electrocardiol 2009; 14:319-326.

$\checkmark 18$ Berry RB, Budhiraja R, Gottlieb DJ, et al: Rules for scoring respiratory events in sleep: update of the 2007 AASM Manual for the
Scoring of Sleep and Associated Events. Deliberations of the Sleep Apnea Definitions Task Force of the American Academy of Sleep Medicine. J Clin Sleep Med 2012;8:597-619.

19 Sleep-related breathing disorders in adults: recommendations for syndrome definition and measurement techniques in clinical research. The Report of an American Academy of Sleep Medicine Task Force. Sleep 1999;22: 667-689.

20 Lang RM, Bierig M, Devereux RB, et al: Recommendations for chamber quantification: a report from the American Society of Echocardiography's Guidelines and Standards Committee and the Chamber Quantification Writing Group, developed in conjunction with the European Association of Echocardiography, a branch of the European Society of Cardiology. J Am Soc Echocardiogr 2005;18:14401463.

21 Devereux RB, Alonso DR, Lutas EM, et al: Echocardiographic assessment of left ventricular hypertrophy: comparison to necropsy findings. Am J Cardiol 1986;57:450-458.

22 Mancia G, Fagard R, Narkiewicz K, et al: 2013 ESH/ESC Guidelines for the management of arterial hypertension: the Task Force for the management of arterial hypertension of the European Society of Hypertension (ESH) and of the European Society of Cardiology (ESC). J Hypertens 2013;31:1281-1357.

23 Harjai KJ, Scott L, Vivekananthan K, et al: The Tei index: a new prognostic index for patients with symptomatic heart failure. J Am Soc Echocardiogr 2002;15:864-868.

24 Bruch C, Schmermund A, Dagres N, et al: TeiIndex in coronary artery disease-validation in patients with overall cardiac and isolated diastolic dysfunction. Z Kardiol 2002;91:472480.

25 Canga A, Kocaman SA, Durakoglugil ME, et al: Relationship between fragmented QRS complexes and left ventricular systolic and diastolic functions. Herz 2013;38:665-670.

26 Yan GH, Wang M, Yiu KH, et al: Subclinical left ventricular dysfunction revealed by circumferential 2D strain imaging in patients with coronary artery disease and fragmented QRS complex. Heart Rhythm 2012;9:928935.

27 Terho HK, Tikkanen J, Junttila J, et al: Prevalence and long-term prognosis of fragmented QRS in standard 12-lead electrocardiogram in middle-aged subjects (abstract). Cirulation 2012;126:A17162.

28 Tei C, Ling LH, Hodge DO, et al: New index of combined systolic and diastolic myocardial performance: a simple and reproducible measure of cardiac function - a study in normals and dilated cardiomyopathy. J Cardiol 1995; 26:357-366.

29 Karatzis EN, Giannakopoulou AT, Papadakis JE, et al: Myocardial performance index (Tei index): evaluating its application to myocardial infarction. Hellenic J Cardiol 2009;50: 60-65. 